\title{
Role of Plasma Soluble Lectin-Like Oxidized Low-Density Lipoprotein Receptor-1 and microRNA-98 in Severity and Risk of Coronary Artery Disease
}

\author{
Md Sayed Ali Sheikh @
}

Department of Internal Medicine, Cardiology, College of Medicine, Jouf University, Sakaka, Aljouf, Kingdom of Saudi Arabia

Background: Coronary artery diseases are the most important cause of premature death, and these diseases are predominantly related to atherosclerosis. Soluble lectin-like oxidized low-density lipoprotein receptor-1 and microRNAs are closely associated with atherosclerotic coronary heart diseases.

Aims: To investigate the relationship between the severity and risk of coronary artery disease and plasma soluble lectin-like oxidized low-density lipoprotein receptor-1 and miR-98.

Study Design: Case-control study.

Methods: Angiographically documented patients with 38 single-vessel, 75 double-vessel, and 62 multi-vessel coronary artery disease; 62 healthy control participants; and 24 -hour hypoxic ( $1 \%$ oxygen) human umbilical vein endothelial cells were included in this study. Circulating soluble lectin-like oxidized low-density lipoprotein receptor-1 concentrations were determined through enzyme-linked immunosorbent assays, and miR-98 expressions were measured by quantitative real-time polymerase chain reaction.

Results: The expressions of plasma soluble lectin-like oxidized low-density lipoprotein receptor-1 levels were progressively and significantly higher in patients with single-vessel, double-vessel, and multi-vessel coronary artery disease than in healthy controls $(\mathrm{p}<0.001)$. Circulating soluble lectin-like oxidized low-density lipoprotein receptor- 1 concentrations in female patients with multi-vessel, double-vessel, and single-vessel coronary artery disease had evidently elevated com- pared with that in male patients $(\mathrm{p}<0.001)$. Plasma soluble lectin-like oxidized low-density lipoprotein receptor-1 levels were remarkably increased in females with coronary artery disease in different age groups compared with the males in the same age groups $(\mathrm{p}<0.001)$. Patients with single-vessel (areas under the curve $=0.879$ ), double-vessel (area under the curve $=0.928$ ), and multi-vessel (area under the curve $=0.943$ ) coronary artery disease have been clearly differentiated from healthy participants with respect to high sensitivity and specificity. The expression of miR-98 was noticeably downregulated in patients with single-, double- and multi-vessel occluded coronary artery disease and in hypoxic human umbilical vein endothelial cell compared with controls $(p<0.001)$. Significantly elevated lectin-like oxidized low-density lipoprotein receptor-1 and caspase- 3 activity and remarkably decreased cellular viability in hypoxic injured human umbilical vein endothelial cell. On the contrary, mimic of miR-98 markedly reduced caspase- 3 and lectin-like oxidized low-density lipoprotein receptor-1 levels and highly increased cellular viability.

Conclusion: Elevated circulating plasma soluble lectin-like oxidized low-density lipoprotein receptor-1 levels have a potential impact to identify the severity of coronary artery disease and have a strong correlation with aging as well as the female gender. Reduced plasma miR98 level is possibly considered a risk factor for coronary artery disease, and agomiR-98 prevents atherosclerosis and cellular injury by targeting lectin-like oxidized low-density lipoprotein receptor-1.
Globally, coronary atherosclerotic heart diseases are the major epidemic health problem and the predominant cause of increased morbidity and mortality. Early identification with proper management of stable coronary artery disease (CAD) may significantly reduce further coronary atherosclerotic complications, including unstable CAD, myocardial infarction, and markedly reduced cardiac mortality rate (1). The clinical presentations of stable CAD especial- ly in younger and geriatric people and in patients with diabetes mellitus and neuropathies are atypical, and sometimes they do not have any clinical symptoms. A large number of patients with stable CAD may have normal electrocardiogram findings, and exercise tolerance test reports may not be significant. Cardiac computed tomography angiogram and especially invasive coronary angiogram may help to confirm the diagnosis of patients with stable CAD,

Address for Correspondence: Md Sayed Ali Sheikh, Department of Internal Medicine, Cardiology, College of Medicine, Jouf University, Sakaka, Aljouf, Kingdom of Saudi Arabia e-mail: drsheikh07@hotmail.com

Received: July 3, 2020 Accepted: September 1,2020 • DOI: 10.5152/balkanmedj.2021.20243

Available at www.balkanmedicaljournal.org

ORCID iD of the author: M.S.A.S. 0000-0001-7187-6550

Cite this article as:

Sheikh MSA. Role of Plasma Soluble Lectin-Like Oxidized Low-Density Lipoprotein Receptor-1 and microRNA-98 in Severity and Risk of Coronary Artery Disease. Balkan Med J 2021;38:13-22

Copyright@Author(s) - Available online at http://balkanmedicaljournal.org/ 
but they require special arrangements and may produce simple to severe complications and may also not be suitable for all patients. Therefore, blood-based biomarkers are necessary for the diagnosis as well as the grading of patients with stable CAD $(1,2)$.

Coronary atherosclerosis is a chronic multifactorial inflammatory process within the coronary arteries. Lectin-like oxidized low-density lipoprotein receptor-1 (LOX-1) plays critical roles in the development of atherosclerosis through binding, internalization, and degradation with oxidized low-density lipoprotein cholesterol (oxLDL) in the arterial wall (3). The human LOX-1, that is, OLR-1, is a $50 \mathrm{kDa}$ transmembrane glycoprotein located in chromosome 12 as a C-type lectin gene cluster. Initially, it was detected in bovine aortic endothelial cells subsequently identified from human coronary artery endothelial cells, cardiomyocytes, macrophages, fibroblasts, platelets, and smooth muscle cells. The extracellular neck area of the LOX-1 protein usually cleaved through an enzymatic reaction (metalloproteinase, ADAM10) and generated soluble LOX-1 (sLOX-1) that circulated in different body fluids, including blood, and triggered release of many inflammatory cytokines and progress to atherosclerosis. This circulatory sLOX-1 has a potential value in diagnosis and prognosis of many cardiovascular diseases (4).

An early stage of the atherosclerosis process mainly initiated by endothelial dysfunction and LOX-1-ox-LDL is primarily involved in vascular injury caused by several inflammatory mechanisms. Activated LOX-1 significantly increased ROS production, endothelial cellular apoptosis, caspase-3, caspase-9, and inhibition of antiapoptotic BCL-2 protein (5). Moreover, PCSK9 and LOX-1 concentrations were elevated in human endothelial cells and vascular smooth muscle cells, and they were significantly linked with ox-LDL and lipopolysaccharide. All these lead to arterial wall damage, a hallmark of atherosclerosis (6). Several clinical studies have demonstrated that circulating sLOX-1 levels were significantly higher in patients with essential hypertension, type 2 diabetes mellitus, metabolic syndrome, heart failure, ischemic heart disease, and ischemia-reperfusion injury $(4,7,8)$. Recently, Valter's research group demonstrated that circulating sLOX-1 concentrations remarkably increased in stable CAD and have a considerable impact on the diagnosis of patients with stable CAD (9). It has also been reported that elevated circulating sLOX-1 levels were positively associated with lesions of stable CAD, acute coronary syndrome, and traditional risk factors of $\operatorname{CAD}(10,11)$.

Furthermore, microRNAs (miRNAs) are highly conserved, endogenous, single-stranded noncoding RNAs that generally regulate gene expression at the posttranscriptional level through the 3 '-untranslated region. It has been well established that miR-217, miR-92, and miR-126 significantly regulated the atherosclerosis process (12). The expressions of circulatory miRNAs (miR-21, miR-149, miR-378, and miR-765) were remarkably altered in patients with $\operatorname{CAD}(13,14)$. Besides, miR-590, miR-155, and miR-let-7g-3p critically regulated LOX-1-ox-LDL-mediated lipid metabolism and prevented human umbilical vein endothelial cell (HUVEC) damage during hypoxic in- jury by modifying their target proteins $(4,15)$. Recently demonstrated miR-98 significantly regulated foam cell formation and lipid accumulation by targeting LOX-1 in mice model; besides, it has modulated various inflammatory cytokines production such as interleukin (IL)6 , IL-8, IL-10, and tumor necrosis factor (TNF)- $\alpha$ in human peripheral blood mononuclear cells $(16,17)$. However, the severity of coronary disease in patients and its correlation with circulating sLOX-1 and miR-98 have not yet been fully investigated. Therefore, this study demonstrated the clinical impact of plasma sLOX-1 level in patients with single-vessel, double-vessel, and multi-vessel stable CAD and linked it with gender and aging. In addition, the role of miR-98 in patients with CAD and its effect on LOX-1 expressions during hypoxic injured HUVECs were also examined.

\section{MATERIALS AND METHODS}

\section{Human study participants}

This study recruited 38 patients with angiographically confirmed single-vessel CAD, 75 double-vessel CAD and 62 patients with multi-vessel CAD and 62 healthy participants from January 2016 to December 2018. Notably, three interventional cardiologists performed the invasive coronary angiogram through radial approach. Coronary arteries occluded at $50 \%$ or more than $50 \%$ were considered CAD. Patients with only one major coronary artery (left anterior descending, left circumflex artery, right coronary artery) blocked were considered to be suffering from single-vessel CAD, those with two major coronary arteries or with left main coronary artery blocked were considered to be suffering from double-vessel $\mathrm{CAD}$, and those with two or more than two major coronary arteries blocked (including stenosis of their diagonal or other branches) were considered to be suffering from multi-vessel CAD. Patients with previously implanted coronary artery stent, history of acute myocardial infarction and stroke, malignancy, chronic obstructive pulmonary disease (COPD), and bronchial asthma were excluded from this study. Patients without cardiovascular diseases and evidence of lipid disorders, chronic inflammatory conditions, and chronic hepatic and renal disorders were included as a healthy participant. A written informed consent was taken from all the participants, and their baseline, clinical, and medication data were collected from the medical record files following the instructions of the Declaration of Helsinki. The patient's $4 \mathrm{~mL}$ blood samples were obtained from an antecubital vein after 72 hours of coronary angiography. In accordance with company's instructions, plasma sLOX-1 levels were measured from all the human samples by enzyme-linked immunosorbent assays (Bio-Rad Laboratories, Hercules, CA, USA). Laboratory parameters, including total cholesterol (T-CHOL), low-density lipoprotein (LDL), triglycerides (TGs), high-density lipoprotein (HDL), creatinine, and high-sensitivity C-reactive protein (hs-CRP) were demonstrated using standard laboratory methods by an automatic biochemical analyzer machine (Hitachi75, Tokyo, Japan).

\section{Cell culture and transfections}

HUVECs were obtained (Chinese Academy of Medical Sciences, Shanghai, China) and harvested using Dulbecco's Modified Eagle's 
Medium (Gibco, Waltham, MA, USA) mixed with 20\% fetal calf serum (HyClone, Logan, UT, USA) in 6-well plates at a density of $5 \times 10^{5}$ cells/well with $95 \%$ humidified atmosphere at $37^{\circ} \mathrm{C}$ in $5 \%$ carbon dioxide $\left(\mathrm{CO}_{2}\right)$. Hypoxic HUVECs were generated by 18 -hour incubation in $1 \%$ oxygen, $95 \%$ nitrogen, and $5 \% \mathrm{CO}_{2}$ using a hypoxic modulator (Model 3131; Forma Scientific, Inc, Marietta, OH, USA) at $37^{\circ} \mathrm{C}$. HUVECs were transfected with has-mimic-miR-98(50 $\mathrm{nmol} / \mathrm{L}$ ) for overexpression and has-mimic-negative control (NC)miR-98 (50 nmol/L) (RiboBio, Guangzhou, China) for serving as an inner control with using Lipofectamine 2000 Transfection Reagent (Invitrogen, Carlsbad, CA, USA) by following the company's instructions; more details are given in our previous study (15).

\section{RNA extraction and measurement of miR-98 levels by quanti- tative real-time polymerase chain reaction}

RNA was extracted from human plasma, normoxic, hypoxic, and transfected cultured HUVECs by using the TRizol reagent (Invitrogen, Carlsbad, CA, USA) following the manufacturer's guidelines. The expression of miR-98 was measured by the 7300 Quantitative Real-Time Polymerase Chain System (Applied Biosystems, Foster City, CA, USA) using Takara SYBR Green Master Mix reagents (Takara, Dalian, China). MiR-98, endogenous microRNA-156a primers structured were obtained from RiboBio, and LOX-1 and $\beta$-actin (inner control) primers were designed by the Sangon Biotech (Shanghai, China); the methods were explained in our previous study (15).

\section{Measurement of caspase-3 activity and cellular viability}

Normal, hypoxic, and transfected HUVECs caspase-3 activity was demonstrated by assay kits (Beyotime, Shanghai, China), and cellular viability was measured by CCK-8 kits (Beyotime, Shanghai, China) following the company's instructions. Briefly, $10 \mu \mathrm{L}$ of cell lysates was added with $90 \mu \mathrm{L}$ of work solution containing caspase-3 substrate (Ac-DEVD-pNA) and subsequently incubated at $37^{\circ} \mathrm{C}$ for 2 hours. Notably, 96-well microplates were used for HUVECs, and each plate absorbance was measured at $405 \mathrm{~nm}$ for the caspase- 3 activity and at $450 \mathrm{~nm}$ for the cellular viability by the SpectraMax reader (Molecular Devices, San Jose, CA, USA).

\section{Statistical analysis}

Normality distributions of the numeric variables data were analyzed by the Shapiro-Wilk test. Continuous variables data were measured as mean with standard deviation, and categorical variables data were expressed as numbers and percentages. The differences between two groups were conducted with Student's $t$ test for normally distributed data, Mann-Whitney $U$ test for abnormally distributed data, and one-way analysis of variance method followed by Tukey post hoc test for multiple groups. Categorical variables were determined through the Chi-square test. The medium effect size was 0.05 , the $\alpha$ error was 0.05 , the power of the study was $90 \%$, and the required total sample size was 192 . Sensitivity and specificity were explored by the receiver operator characteristic (ROC) curve. All p values were two sided, and $\mathrm{p}<0.05$ was considered statistically significant. IBM Statistical Package for Social Sciences for Windows version 20 was used for analyzing all the data (IBM SPSS Corp.; Armonk, NY, USA).

\section{RESULTS}

Baseline and clinical information of the study participants This study involved 38 patients with single-vessel CAD, 75 patients with double-vessel CAD, and 62 patients with multi-vessel CAD and 62 healthy study participants. The characteristics of the study participants are presented in Table 1. Body mass index (BMI), family history of CAD, and hs-CRP were significantly changed between controls and patients with single-vessel, double-vessel, and multi-vessel CAD $(p<0.001)$ but were not statistically significant in patients with single-vessel, double-vessel, and multi-vessel CAD. T-CHOL and HDL concentrations in patients with double-vessel and multi-vessel CAD were significantly higher than healthy participants, whereas TG and LDL levels were significantly different among healthy participants and patients with multi-vessel CAD $(\mathrm{p}<0.001)$.

\section{Plasma sLOX-1 concentrations in healthy participants and in patients with coronary artery occluded CAD}

The plasma sLOX-1 expressions were significantly elevated by 6.6-fold in patients with multi-vessel CAD $(632 \pm 54 \mathrm{pg} / \mathrm{mL})$, by 4.4-fold in patients with double-vessel CAD $(418 \pm 51 \mathrm{pg} / \mathrm{mL})$, and by 3.8 -fold in patients with single-vessel CAD $(367 \pm 40 \mathrm{pg} / \mathrm{mL})$ compared with healthy participants $(95 \pm 12 \mathrm{pg} / \mathrm{mL})(\mathrm{p}<0.001)$. The pattern of sLOX-1 expressions within patients with single-vessel, double-vessel, and multi-vessel CAD lesions were highly different $(p<0.001)$ (Figure 1a). Furthermore, female patients with single-vessel, double-vessel, and multi-vessel occluded CAD had markedly higher plasma sLOX-1 levels than males, indicating that circulating sLOX-1 concentrations were significantly linked with female patients with CAD (Figure 1b). Besides, circulatory plasma sLOX-1 expressions in healthy female participants were slightly higher than healthy male participants but were not significant.

\section{The relationship of plasma sLOX-1 expression with age variations in patients with CAD lesions and in control participants}

Plasma sLOX-1 levels were evidently increased in female patients (aged 29-49 years, 50-69 years, and 70-80 years) with multi-vessel CAD lesions compared with that in male patients in the same age groups with multi-vessel CAD lesions ( $\mathrm{p}<0.001$ ), whereas circulating sLOX-1 were not significant within male participants in different age groups; it is suggested that female patients with multi-vessel CAD were associated with sLOX-1 concentrations (Figure 2a). Female patients with double-vessel CAD (aged 50-69 years and 70-80 years) had significantly higher plasma sLOX-1 expression than male patients in the same age groups with double-vessel occluded CAD $(\mathrm{p}<0.001)$; both female and male patients with double-vessel CAD (aged 29-49 years) showed no significant difference (Figure 2c). The expression of circulating sLOX-1 concentrations in female patients (aged 29-49 years and 50-69 years) with single-vessel CAD were noticeably upregulated compared with male patients in similar age groups with single-vessel CAD $(\mathrm{p}<0.001)$ (Figure $2 \mathrm{c})$. Moreover, sLOX-1 concentrations were slightly increased in both healthy male and female participants among all the age groups (Figure $2 \mathrm{~d}$ ). 
TABLE 1. Clinical parameters of the study groups

\begin{tabular}{|c|c|c|c|c|c|c|c|}
\hline Characteristic & Healthy participants & Single-vessel CAD & Double-vessel CAD & Multi-vessel CAD & $\mathrm{P} 1$ & $\mathrm{P} 2$ & P3 \\
\hline Sample size & 62 & 38 & 75 & 62 & & & \\
\hline Age, y & $54.7 \pm 10.8$ & $61.93 \pm 11.5$ & $63.95 \pm 11.9$ & $66 \pm 12.7$ & 0.256 & 0.197 & 0.143 \\
\hline Gender, male/female & $33 / 29$ & $22 / 16$ & $41 / 34$ & $33 / 29$ & 0.688 & 0.867 & 1.000 \\
\hline BMI, $\mathrm{kg} / \mathrm{m}^{2}$ & $22.7 \pm 2.8$ & $23.9 \pm 4.6$ & $25.7 \pm 5.1$ & $25.9 \pm 8.7$ & 0.128 & 0.000 & 0.000 \\
\hline \multicolumn{8}{|l|}{$\mathrm{CAD}$ risk factors, $\%$} \\
\hline Tobacco smoker & $61(37)$ & $69(26)$ & $71(53)$ & $76(47)$ & 0.403 & 0.208 & 0.083 \\
\hline Hypercholesterolemia & 0 & $83(31)$ & $86(64)$ & $88(54)$ & - & - & - \\
\hline Type 2 diabetes mellitus & 0 & $21(8)$ & $30(22)$ & $33(20)$ & - & - & - \\
\hline Family history of CAD & $8(5)$ & $57(21)$ & $61(45)$ & $64(39)$ & 0.000 & 0.000 & 0.000 \\
\hline Hypertension & 0 & $71(27)$ & $75(56)$ & $81(50)$ & - & - & - \\
\hline \multicolumn{8}{|l|}{ Investigation data } \\
\hline $\begin{array}{l}\text { High-sensitivity C-reactive } \\
\text { protein, } \mathrm{mg} / \mathrm{L}\end{array}$ & $1.92 \pm 0.35$ & $14.2 \pm 6.17$ & $18.5 \pm 9.41$ & $22.7 \pm 11.86$ & 0.000 & 0.000 & 0.000 \\
\hline Creatinine $(\mu \mathrm{mol} / \mathrm{L})$ & $82.5 \pm 17.41$ & $86.4 \pm 28.13$ & $89.2 \pm 35.19$ & $89.81 \pm 37.6$ & 0.773 & 0.426 & 0.215 \\
\hline T-CHOL $(\mathrm{mmol} / \mathrm{L})$ & $3.59 \pm 0.04$ & $5.36 \pm 3.26$ & $6.97 \pm 1.85$ & $8.28 \pm 7.13$ & 0.094 & 0.000 & 0.000 \\
\hline $\mathrm{TG}(\mathrm{mmol} / \mathrm{L})$ & $1.53 \pm 0.62$ & $2.05 \pm 0.49$ & $2.86 \pm 0.64$ & $3.95 \pm 0.82$ & 0.118 & 0.072 & 0.000 \\
\hline $\mathrm{LDL}(\mathrm{mmol} / \mathrm{L})$ & $2.57 \pm 0.81$ & $3.95 \pm 1.25$ & $4.22 \pm 1.07$ & $5.16 \pm 1.88$ & 0.217 & 0.094 & 0.000 \\
\hline $\mathrm{HDL}(\mathrm{mmol} / \mathrm{L})$ & $1.33 \pm 0.56$ & $0.92 \pm 0.17$ & $0.74 \pm 0.52$ & $0.23 \pm 0.69$ & 0.310 & 0.000 & 0.000 \\
\hline Left ventricular EF \% & $61.25 \pm 11.17$ & $59.33 \pm 8.31$ & $54.87 \pm 6.27$ & $49.87 \pm 4.53$ & 0.479 & 0.281 & 0.064 \\
\hline \multicolumn{8}{|l|}{ Medications, $\%$} \\
\hline Antiplatelet agents & 0 & $76(29)$ & $89(67)$ & $92(57)$ & - & - & - \\
\hline Beta blockers & 0 & $81(31)$ & $85(64)$ & $87(54)$ & - & - & - \\
\hline Nitrates groups & 0 & $64(24)$ & $73(55)$ & $77(48)$ & - & - & - \\
\hline Calcium blockers & 0 & $7(3)$ & $10(7)$ & $14(9)$ & - & - & - \\
\hline Statins drugs & 0 & $68(26)$ & $71(53)$ & $74(46)$ & - & - & - \\
\hline
\end{tabular}

TABLE 2. The ROC curve analysis of plasma sLOX-1 ratios in the prediction of patients with CAD

\begin{tabular}{lccccccc}
\hline Plasma sLOX-1 & AUC & Cutoff point & Sensitivity (\%) & Specificity (\%) & CI (95\%) & Positive value & Negative value \\
\hline $\begin{array}{l}\text { Patients with single-vessel CAD } \\
\text { versus control participants }\end{array}$ & 0.879 & 1.79 & 0.89 & 0.90 & 0.815 to 0.942 & 38 & 62 \\
$\begin{array}{l}\text { Patients with double-vessel CAD } \\
\text { versus healthy participants }\end{array}$ & 0.928 & 1.97 & 0.99 & 0.98 & 0.878 to 0.978 & 75 & 62 \\
$\begin{array}{l}\text { Patients with Multi-vessel CAD } \\
\text { versus control participants }\end{array}$ & 0.943 & 1.89 & 0.96 & 0.94 & 0.896 to 0.989 & 62 & 62 \\
\hline
\end{tabular}

The AUC curve, cutoff point, sensitivity, specificity, positive and negative value, CI (95\%), and p values of sLOX-1 are presented in Table 2.

AUC: area under the curve, CAD: coronary artery disease, CI: confidence interval, ROC: receiver operator curve, sLOX-1: soluble lectin-like oxidized low-density lipoprotein receptor-1.

\section{Impact of sLOX-1 in the severity of patients with CAD}

The clinical impact of plasma sLOX-1 to identify the degree of coronary artery stenosis was evaluated through the ROC curve. Circulating sLOX-1 expressions in patients with single-vessel CAD lesions were strongly distinguished from healthy participants with high specificity and sensitivity (Table 2) with an area under the curve (AUC) of 0.879 (Figure 3a). In patients with double-vessel CAD, sLOX-1 levels were evidently differentiat- ed from healthy participants with remarkable specificity and sensitivity, and the AUC was 0.928 (Figure 3b). The ROC curve of circulating plasma sLOX-1 between controls and patients with multi-vessel CAD revealed a significant discrimination with a prominent AUC of 0.943 (Figure 3c). Moreover, ROC curves of plasma sLOX-1 were also highly significant and able to discriminate among patients with single-vessel, double-vessel, and multi-vessel CAD. These findings recommended that upregulat- 
a

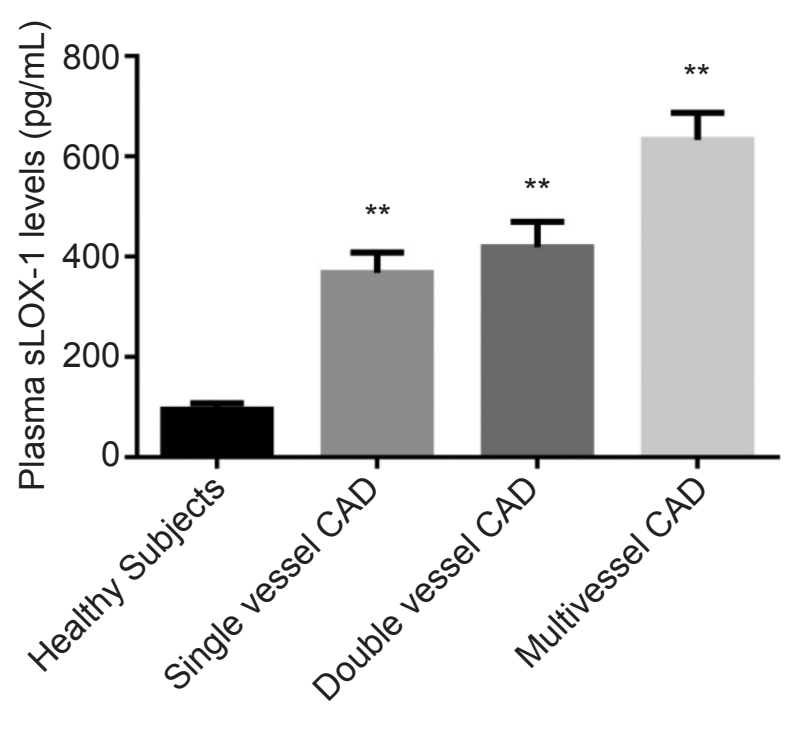

b

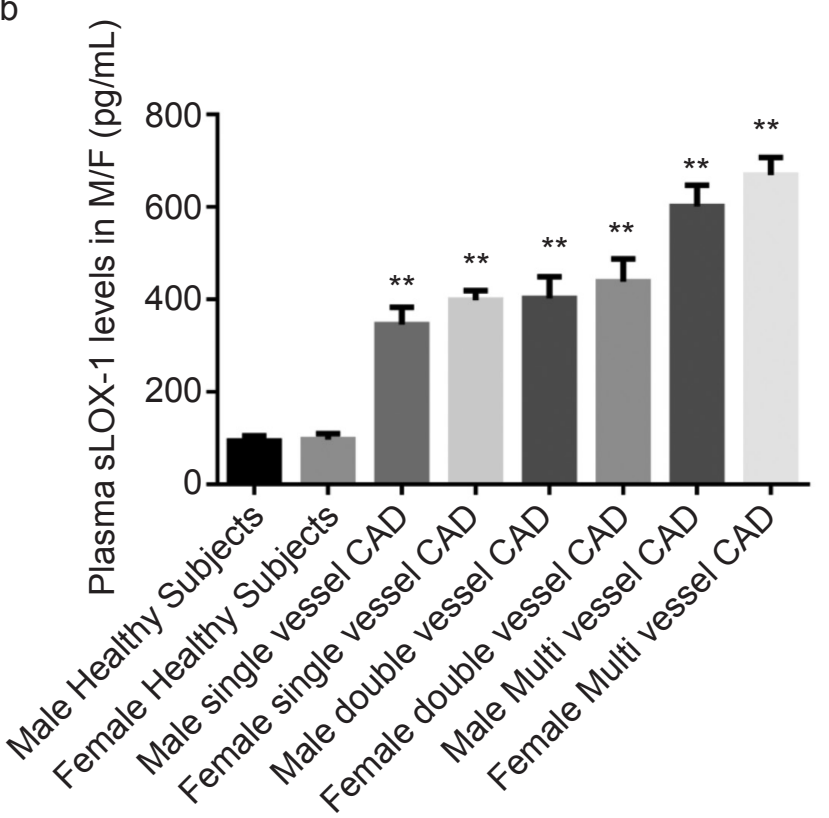

FIG. 1. a, b. The expression of circulating SLOX-1 between healthy participants and patients with single-vessel, double-vessel, and multi-vessel CAD (a). The relative expression of plasma sLOX-1 in males and females among different patients with CAD lesions and also in healthy participants. Plasma sLOX-1 was evaluated by ELISA technique and quantitative data were expressed as mean $\pm S D$ from three independent experiments (b). ${ }^{* *} p<0.001$. CAD: coronary artery disease, ELISA, enzyme-linked immunosorbent assay, SD: standard deviation, sLOX-1: soluble lectin-like oxidized low-density lipoprotein receptor-1

ed plasma sLOX-1 has an essential impact on categorizing the severity of coronary artery stenosis.

Expression of miR-98 in the human plasma and HUVECs Circulating human plasma miR-98 expressions were significantly and progressively lower in patients with single-vessel, double-vessel, and multi-vessel CAD lesions than that in the healthy control groups $(\mathrm{p}<0.001)$. Besides, plasma miR-98 concentrations in patients with multi-vessel CAD were remarkably downregulated compared with patients with double-vessel and single-vessel CAD patients (Figure 4a). Moreover, miR-98 expressions were evidently reduced in 18-hour hypoxic incubated HUVECs than normal HUVECs $(\mathrm{p}<0.001)$ (Figure 4b).

\section{Effect of miR-98 in LOX-1, Caspase-3, and cellular viability in hypoxic induced HUVECs}

LOX-1 is the direct target gene of miR-98 identified from the target scan. The expressions of LOX-1 mRNA protein were significantly elevated in 18-hour hypoxic induced HUVECs compared with normal HUVECs, but LOX-1 expressions were amazingly reduced compared with hypoxic HUVECs transfected with mimic-miR98 $(p<0.001)$ (Figure 5a). The relative activities of caspase-3 in 18hour hypoxic injured HUVECs were noticeably higher than normoxic HUVECs. Conversely, caspase-3 activities in 18-hour hypoxic HUVECs were markedly downregulated with overexpression of miR-98 $(\mathrm{p}<0.001)$, and $\mathrm{NC}$ showed no obvious reaction with hypoxic groups (Figure $5 \mathrm{~b}$ ). Cellular viability markedly re- duced in hypoxic conditions, but HUVECs viability distinctly increased with mimic-miR-98 (Figure 5c).

\section{DISCUSSION}

CAD usually occurs because of the formation of an atherosclerotic plaque within the coronary artery. Increased plasma sLOX-1 levels are considered an early predictor of endothelial injury and atherosclerotic plaque (7). This study found that plasma sLOX1 levels had significantly higher expressions in patients with multi-vessel CAD followed by patients with double-vessel and single-vessel CAD compared with healthy controls. Moreover, circulating sLOX-1 expressions within patients with single-vessel, double-vessel, and multi-vessel CAD were also highly significant. Besides, sLOX-1 concentrations in female patients with single-vessel, double-vessel, and multi-vessel CAD were noticeably elevated compared with that seen in male patients. These results suggested plasma SLOX-1 concentrations have a significant role to differentiate patients with simple and complex CAD, and its level may be potentially useful to determine the progression of CAD lesions. Zhao et al. (18) demonstrated that patients with complex CAD lesions had remarkable higher circulating serum sLOX1 concentrations than patients with simple CAD lesions, which closely linked with the present clinical research. Research studies reported that sLOX-1 levels are highly expressed in patients with CAD with three to four occluded vessels and also in the proximal segment of LAD lesions compared with that in the distal portion of 
a

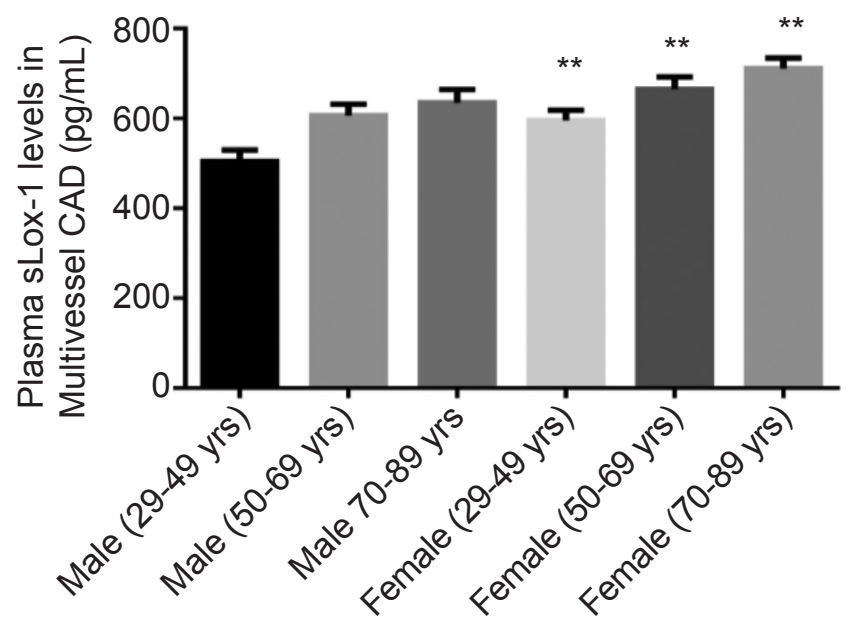

C

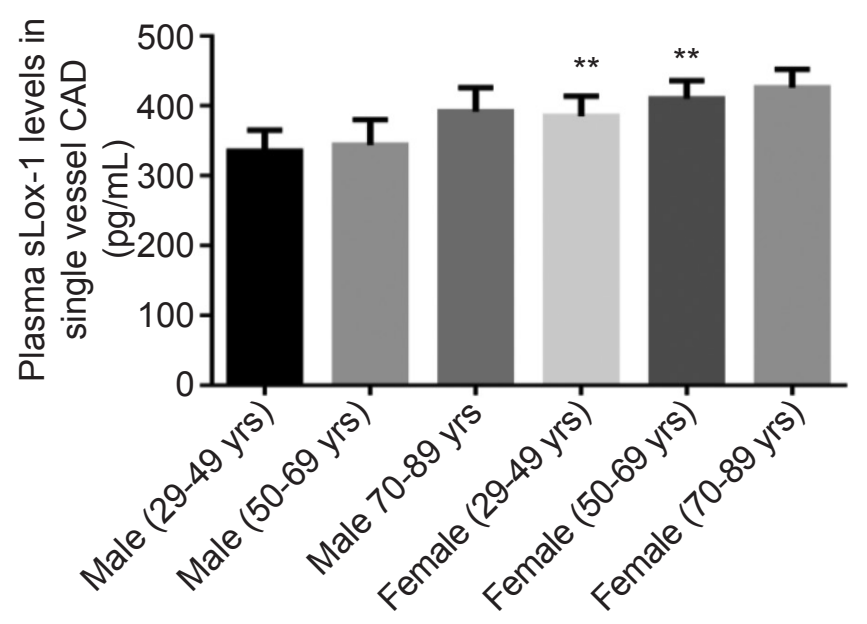

b

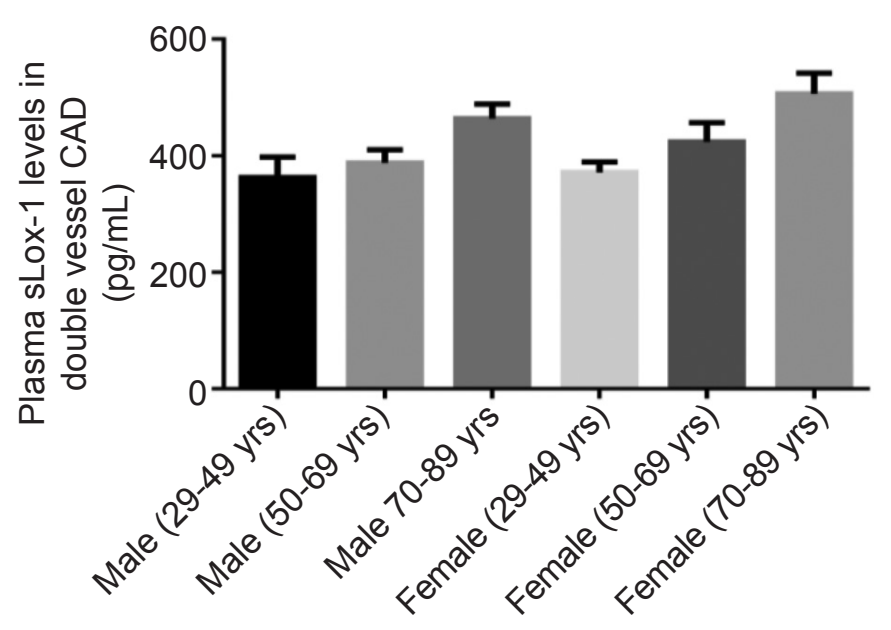

d

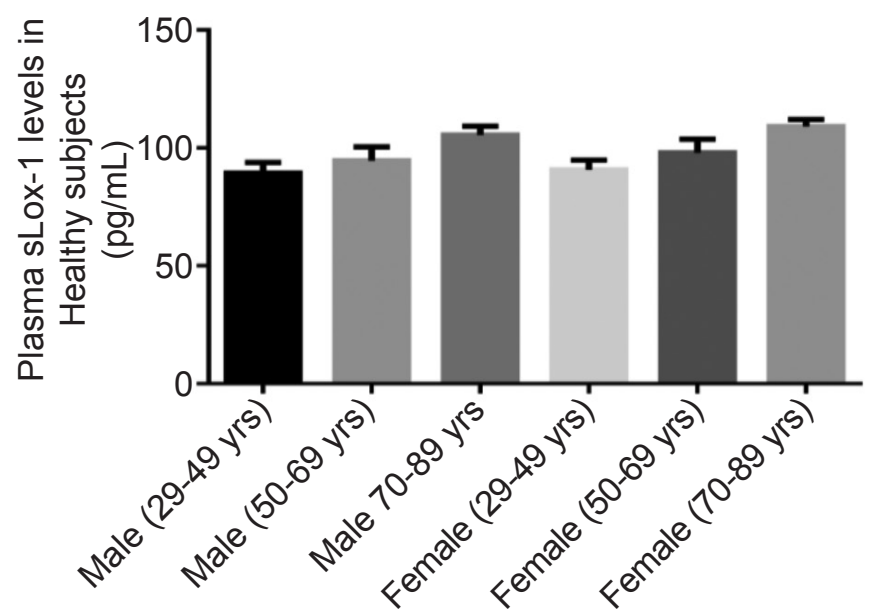

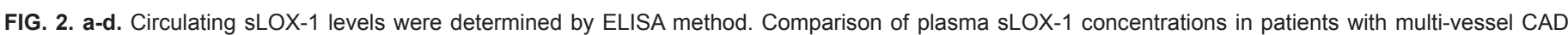

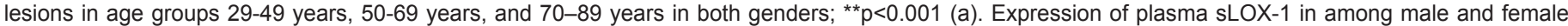

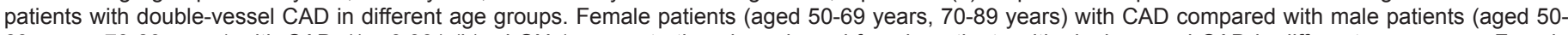

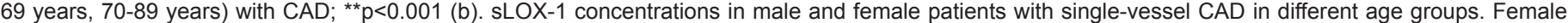

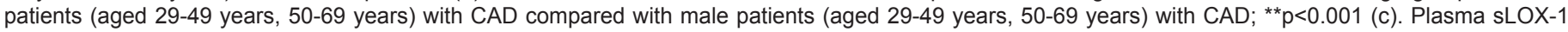

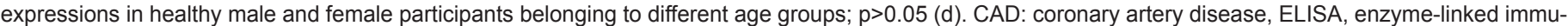
nosorbent assay, sLOX-1: soluble lectin-like oxidized low-density lipoprotein receptor-1

LAD lesions; their results are directly supported by the this study $(19,20)$. The sensitivity and specificity of CAD lesions have been investigated by the ROC curve. The patients with single-vessel, double-vessel, and multi-vessel CAD were clearly differentiated from healthy participants with AUC of $0.879,0.928$, and 0.943 , respectively. The sensitivity and specificity values among patients with single-vessel, double-vessel, and multi-vessel CADs were evidently higher and statistically significant. These findings indicated that upregulated plasma sLOX-1 may be used as a novel clinical parameter for determining the severity of the CAD lesions. These results also were in accordance with other clinical studies $(8,21$, 22). Zhao et al. (23) and Liu et al. (24) in their clinical studies reported that circulating serum SLOX-1 concentrations are signifi- cantly linked with major cardiovascular events, and the concentration may be used as a prognostic marker and risk stratification in patients with stable CAD after percutaneous coronary intervention.

Although age and gender are the major nonmodifiable risk factors for CADs, there is a lack of information regarding sLOX1 and aging. This study for the first time demonstrated sLOX-1 concentrations among different ages between healthy male and female participants and male and female patients with single-vessel, double-vessel, and multi-vessel CAD. Circulating sLOX-1 expressions in healthy female participants in different age groups were fairly higher than healthy male participants. Very interestingly, this research found that circulating sLOX-1 values were 
a
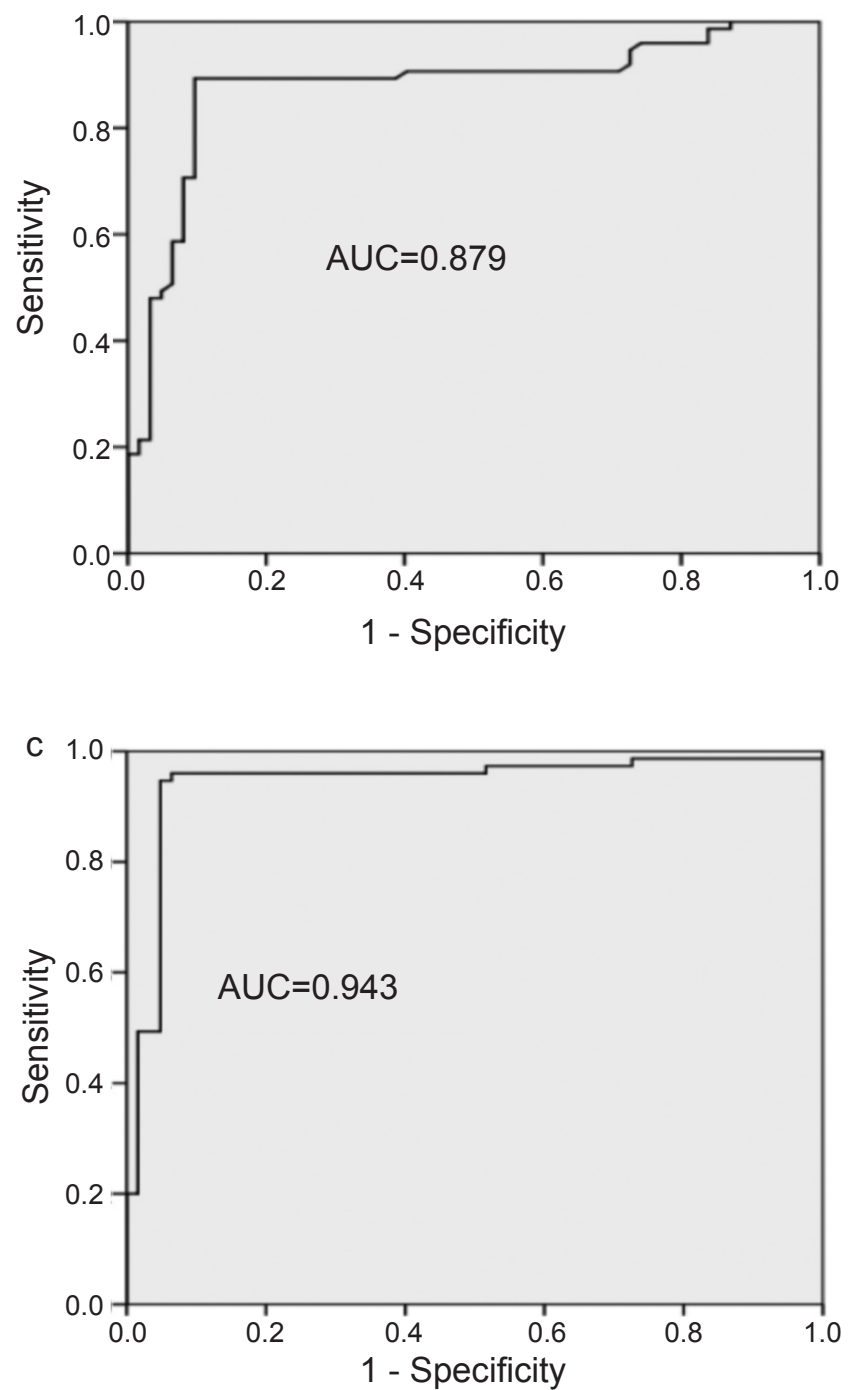

markedly upregulated in female patients (aged 29-49 years, 5069 years) with single-vessel CAD compared with male patients belonging to similar groups, but sLOX-1 expression among female and male patients aged 70-89 years have not been prominently elevated, which may be because of the less number of patients with single-vessel CAD in this age group. Female patients (aged 50-69 years and 70-89 years) with double-vessel CAD had remarkably higher plasma sLOX-1 expression than male patients in the same age groups, whereas female and male patients aged 29-49 years have not shown any obvious difference, probably because double-vessel CAD are usually not very common in this female age group. Circulating plasma sLOX-1 levels in female patients (aged 29-49 years, 50-69 years, and 70-89 years) with multi-vessel CAD were remarkably upregulated compared with male patients in the same age groups. These results strongly supported that sLOX-1 concentrations are evidently associated with the progression of patients with CAD and closely linked with female gender. Andreas et al. (25) identified that sLOX-1 expres-

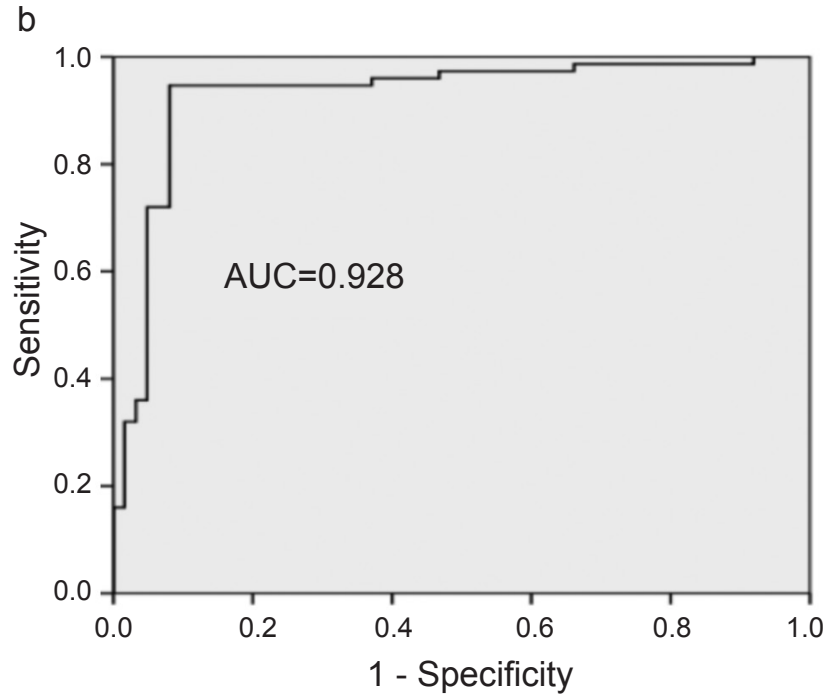

FIG. 3. a-c. ROC curve analysis was applied for discrimination of the severity of CAD lesions in patients with single-vessel, double-vessel, and multi-vessel CAD. Patients with single-vessel CAD and healthy participants $(A \cup C=0.879)$ (a). Comparison between patients with double-vessel CAD and healthy participants $(A \cup C=0.928)(b)$. Control participants and patients with multi-vessel CAD (AUC=0.943) (c). AUC: area under the curve; CAD: coronary artery disease, ROC: receiver operator characteristic.

sions were significantly higher in the wall of epicardial coronary arteries in male patients (median age $=61$ years) with three-vessel CAD, but they did not explore expression pattern in the female gender. The study herein found that highest expression of hs-CRP levels was measured in patients with multi-vessel CAD followed by patients with double-vessel and single-vessel CAD, and they were statistically significant. It is suggested that hs-CRP has a major role in the initiation and progression of CAD lesions. Nicole et al. (26) investigated that LOX-1 positively correlated with CRP through ox-LDL and enhanced endothelial dysfunction during atherosclerosis process. Our previous study also demonstrated that CRP levels were significantly increased in patients with CAD compared with healthy participants (14). In addition, family history of CAD was significantly higher in patients with single-vessel, double-vessel, and multi-vessel CAD than in healthy participants; BMI measurement was distinctly higher in patients with double-vessel and multi-vessel CAD than in healthy participants; TC levels were prominently elevated and HDL re- 
a

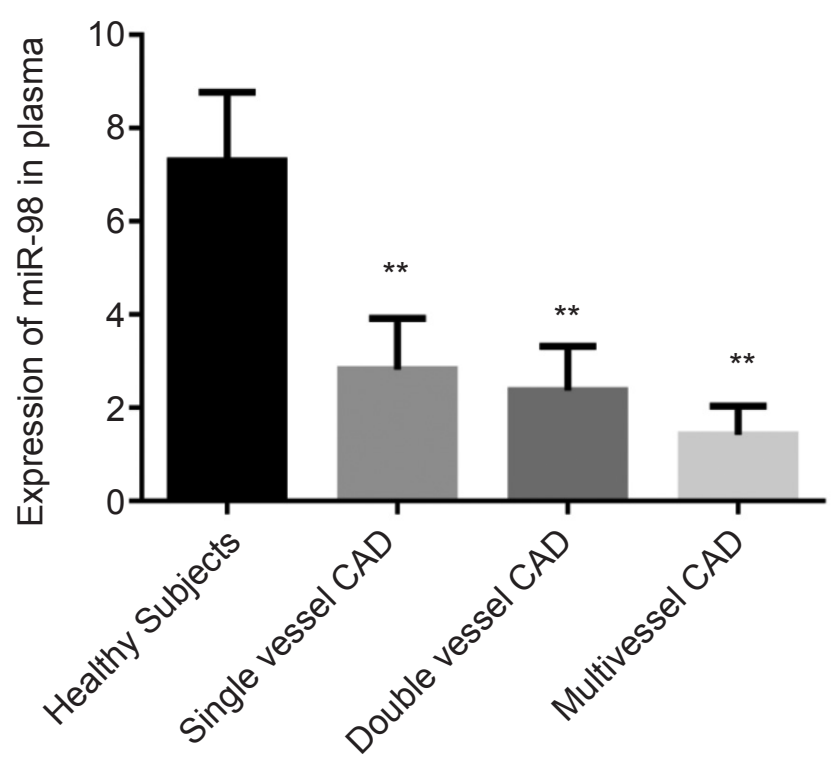

b

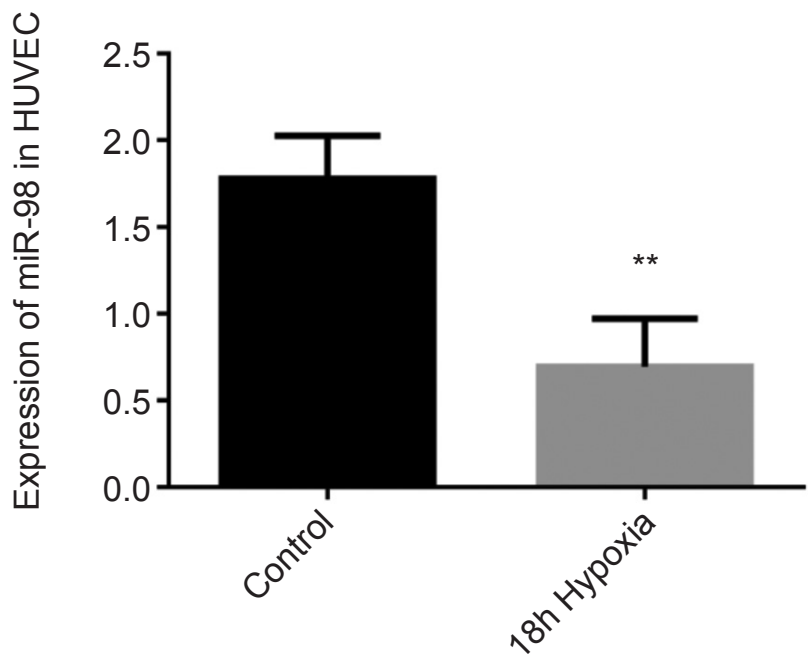

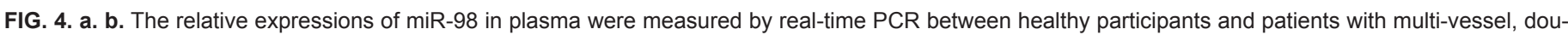

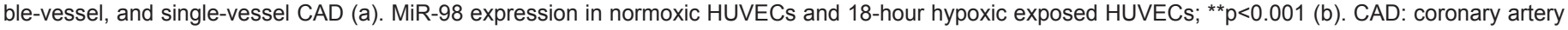
disease, HUVEC: human umbilical vein endothelial cell, PCR: polymerase chain reaction.

markably decreased in patients with double-vessel and multi-vessel CAD compared with that in healthy participants; and TG and LDL levels were obviously increased in patients with multi-vessel CAD compared with that in healthy participants. These data suggested that lipid abnormalities were intimately related with the severity of CAD lesions.

Moreover, miRNAs are significantly involved in various cardiovascular diseases, including CAD. Several circulating miRNAs have a potential utility as diagnostic and prognostic biomarkers for patients with ischemic heart disease (14). A very important finding of this study is that plasma miR-98 was markedly decreased in patients with single-vessel CAD followed by patients with double-vessel and multi-vessel CAD compared with healthy participants. Furthermore, the expressions of miR-98 were significantly downregulated in 18-hour hypoxic exposed HUVECs than in normoxic cell. Recent clinical research demonstrated that serum miR-98 expressions were evidently lower in patients with bronchial asthma than healthy participants (27). However, this research excluded patients with COPD and bronchial asthma to reduce the possible error. This study through TargetScan, MiRFinder, and miRanda bioinformatics databases and also through other studies confirmed that LOX-1 is the direct target protein of miR-98 $(16,28)$. This study revealed that LOX-1 expressions in 18-hour hypoxic injured HUVECs were markedly upregulated compared with that in normoxic cells, whereas overexpression of miR-98 amazingly downregulated LOX-1 expressions as close to normoxic cells; it is recommended that miR-98 significantly controlled LOX-1 expressions during hypoxic damage of HUVECs. Yao dai et al. (16) reported that miR-98 has an essential role to control foam cell formation and prevent excess cholesterol accumulation in ApoE mice through modification of LOX-1 protein expressions. Chen et al. (28) found that overexpression of miR-98 remarkably increased HUVEC viability through regulation of LOX-1 gene expression. The Chuan Sun research group established that agomiR-98 significantly reduced caspase-3 activity and increased cellular viability during ischemic injury (29).

This research found that caspase-3 activity levels were exceptionally increased in 18-hour hypoxic HUVECs compared with that in normal cells; on the contrary, caspase- 3 activities were obviously decreased in mimic-miR-98 hypoxic HUVECs. Furthermore, cellular viability was significantly lower in hypoxic induced HUVECs groups than in normal groups, whereas cellular survivability was remarkably enhanced in hypoxic HUVECs treated with agomiR-98. These results indicated that mimic-miR-98 protects against cellular injury in hypoxic conditions and may be proposed as a therapeutic strategy for coronary atherosclerotic disease. To reduce angiographic dye and catheter effect on vascular injury and circulatory expression of sLOX-1 and miR-98, blood samples were taken after 72 hours of coronary angiography from all patients with confirmed CAD.

Some limitations of this study need to be addressed. Importantly, the data collected from only one provincial tertiary care hospital and the sample sizes among different groups were relatively small. Reporter gene assays have not been performed. Further multicenter with larger study samples are required to establish the circulating sLOX-1 as risk stratification for detecting the severity of coronary heart disease in 
a

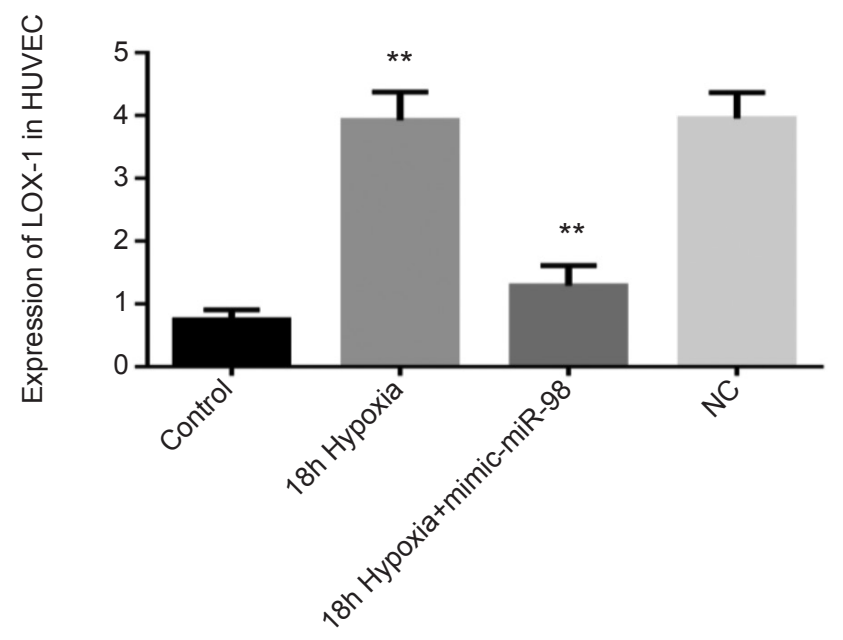

C

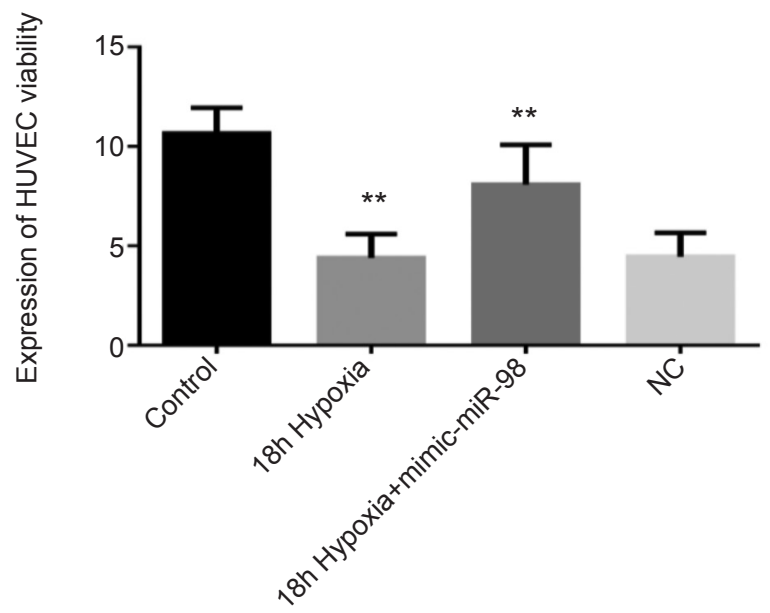

patients. Moreover, with respect to the lipid levels, the relationship between SLOX-1 and miR-98, which were also linked with inflammatory markers such as IL-6, TNF $\alpha$, and hs-CRP, needs to be studied further to discover their underline molecular mechanism.

Circulating sLOX-1 can be used as a potential predictor to recognize the severity of CAD in patients. Reduced plasma miR-98 levels may be considered a risk factor for patients with CAD. Overexpression of miR-98 suppressed caspase-3 activity and improved cellular viability by targeting LOX-1 and providing a possible therapeutic strategy against patients with atherosclerotic CAD.

Ethics Committee Approval: Ethics committee approval was received for this study from the The Xiangya hospital institutional Ethical Committee of Central South University (088110011).

Patient Consent for Publication: A written informed consent was taken from all the participants.

Acknowledgement: The author would like to special thanks to cardiology section of Xiang ya hospital, professor Yang, Xia Ke, and Li fei for their help in this research.

Data-sharing Statement: N/A. b

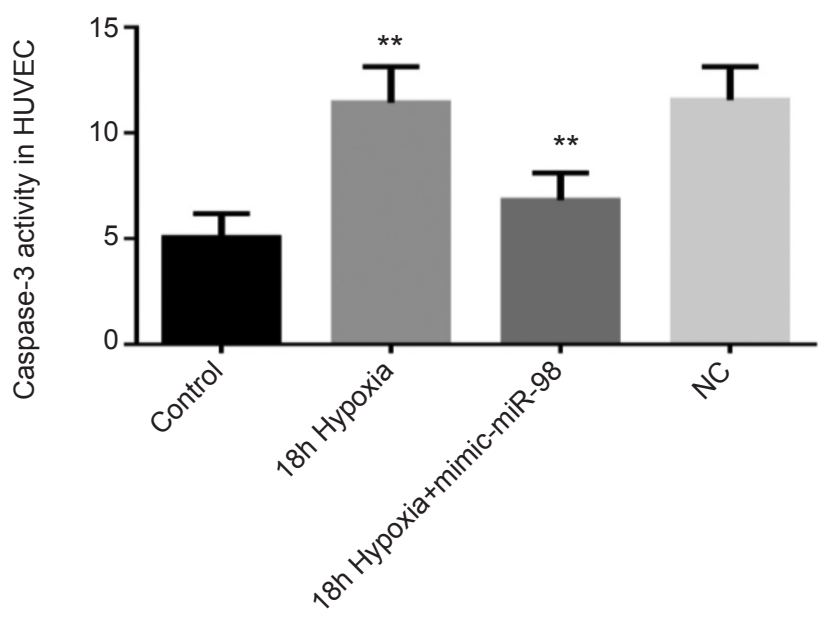

FIG. 5. a-c. Expressions of LOX-1 were detected in normal, 18-hour hypoxic $(1 \%$ O2), 18-hour hypoxic plus transfected with mimic-98 and NC HUVECs by qRT-PCR. Control HUVECs and 18-hour hypoxic HUVECs; ${ }^{* *} p<0.001$. 18-hour hypoxic groups compared with 18-hour hypoxic + mimic-98 HUVECs; ${ }^{* *} p<0.001$. 18-hour hypoxic incubated HUVECs versus NC; $p>0.05$ (a). Caspase-3 activity in agomiR-98 transfected hypoxic HUVECs and hypoxic cell; ${ }^{* *} p<0.001$. Normoxic cells compared with 18-hour hypoxic HUVECs; ${ }^{* *} p<0.001$. 18-hour hypoxic induced HUVECs and NC; $p>0.05$. Caspase-3 activities were measured by the SpectraMax reader (b). Cellular viability between 18-hour hypoxic and miR-98 treated hypoxic HUVECs; ${ }^{* *} p<0.001 .18$ hour hypoxic exposed HUVECs and normoxic condition HUVECs; ${ }^{* *} p<0.001$. NC HUVECs compared with 18-hour hypoxic injured HUVECs; $p>0.05$. The relative expressions of cellular viabilities were calculated through qRT-PCR (c). HUVEC: human umbilical vein endothelial cell, LOX-1: lectin-like oxidized low-density lipoprotein receptor-1, NC: negative control, O2: oxygen, qRTPCR: quantitative real-time polymerase chain reaction.

Conflict of Interest: The authors have no conflicts of interest to declare.

Funding: The authors declared that this study has received no financial support.

\section{REFERENCES}

1. Salim SV, Alvaro A, Emelia JB, Marcio SB, Clifton WC, April PC, et al. Heart Disease and Stroke Statistics-2020 Update: A Report from the American Heart Association. Circulation 2020;141:e139-e596. [Crossref]

2. Juhani K, William W, Antti S, Davide C, Emanuele B, Christian F, et al. 2019 ESC Guidelines for the Diagnosis and Management of Chronic Coronary Syndromes. Eur Heart J 2020;41:407-77. [Crossref]

3. Kunming T, Sayoko O, Peter J L, Suo WX, Tatsuya S. Targeting LOX-1 in Atherosclerosis and Vasculopathy: Current Knowledge and Future Perspectives. Ann N Y Acad Sci 2019;1443:34-53. [Crossref]

4. Ajoe JK, Sri HK, Jawahar LM. Role of Ox-LDL and LOX-1 in Atherogenesis. Curr Med Chem 2019;26:1693-700. [Crossref]

5. Sanjiv S, Avtar SG. Upregulated LOX-1 Receptor: Key Player of the Pathogenesis of Atherosclerosis. Curr Atheroscler Rep 2019;21:38. [Crossref]

6. Zufeng D, Shijie L, Xian W, Xiaoyan D, Yubo F, Jiwani S, et al. Cross-talk Between LOX-1 and PCSK9 in Vascular Tissues. Cardiovasc Res 2015;107:556-67. [Crossref] 
7. Naga VKP, Sotirios KK, Zufeng D, Arockiasamy A, Kottayil IV, Jawahar LM. LOX1 in Atherosclerosis and Myocardial Ischemia: Biology, Genetics, and Modulation. J Am Coll Cardiol 2017;69:2759-68. [Crossref]

8. Martine P, Robert D, Alexis B. Scavenger Receptor LOX1 Genotype Predicts Coronary Artery Disease in Patients with Familial Hypercholesterolemia. Can J Cardiol 2017;33:1312-8. [Crossref]

9. Valter L, Alessandro P, Irene T, Simona S, Serena P, Sergio B, et al. Emerging Biomarkers of Oxidative Stress in Acute and Stable Coronary Artery Disease: Levels and Determinants. Antioxidants (Basel) 2019;8:115. [Crossref]

10. Anja H, Coy B, Steffen W, Christian R, Henning M. Soluble LOX-1: A Novel Biomarker in Patients with Coronary Artery Disease, Stroke, and Acute Aortic Dissection? J Am Heart Assoc 2020;9:e013803. [Crossref]

11. AnSheng L, YuChen W, ShihSheng C, PingHang L, ChiaMing C, Jonathan L, et al. Detection of a High Ratio of Soluble to Membrane-Bound LOX-1 in Aspirated Coronary Thrombi from Patients with ST-Segment-Elevation Myocardial Infarction. J Am Heart Assoc 2020;9:e014008. [Crossref]

12. Alexey C, Volha S, Andrey G, Varvara O, Alexander O. MicroRNAs as Potential Biomarkers in Atherosclerosis. Int J Mol Sci 2019;20:5547. [Crossref]

13. Sri HK, Joseph I, Kameel K, Hongyu G, Yunlong L, Todd CS, et al. Next Generation MicroRNA Sequencing to Identify Coronary Artery Disease Patients at Risk of Recurrent Myocardial Infarction. Atherosclerosis 2018; 278:232-9. [Crossref]

14. Sheikh SA. Diagnostic Role of Plasma MicroRNA-21 in Stable and Unstable Angina Patients and Association with Aging. Cardiol Res Pract 2020;2020:9093151. [Crossref]

15. Lei X, Gang Z, Hong Z, Shijun W, Aijun S, Yunzeng Z, et al. Peroxisome Proliferator-Activated Receptor- $\gamma$ Antagonizes LOX-1-Mediated Endothelial Injury by Transcriptional Activation of miR-590-5p. PPAR Res 2019;2019:2715176. [Crossref]

16. Yao D, Xiaoqin W, Dongsheng D, Jun L, Jawahar LM. MicroRNA-98 Regulates Foam Cell Formation and Lipid Accumulation Through Repression of LOX-1. Redox Biol 2018; 16:255-62. [Crossref]

17. Shiwen Y, Chun T, Dongying C, Fangfei L, Mingcheng H, Jinghua Y, et al. miR-98 Modulates Cytokine Production from Human PBMCs in Systemic Lupus Erythematosus by Targeting IL-6 mRNA. J Immunol Res 2019;2019:9827574. [Crossref]

18. ZiWen Z, XueLi Z, YuKun L, ChaoGui L, LiangLong C. Circulating Soluble Lectin-Like Oxidized Low-Density Lipoprotein receptor-1 Levels Are Associated with Angiographic Coronary Lesion Complexity in Patients with Coronary Artery Disease. Clin Cardiol 2011;34:172-7. [Crossref]
19. Valter L, Serena DT, Giuseppina N, Pietro DC, Giuseppina B. Circulating Levels of Lectin-Like Oxidized Low-Density Lipoprotein receptor-1 Are Associated with Inflammatory Markers. Lipids 2008;43:945-50. [Crossref]

20. Mehmet B, Ahmet C, MA Kobat. Circulating Soluble Lectin-Like Oxidized Low-Density Lipoprotein receptor-1 Levels Are Associated with Proximal/Middle Segment of the LAD Lesions in Patients with Stable Coronary Artery Disease. Clin Res Cardiol 2012;101:247-53. [Crossref]

21. Nobutaka I, Tomonori O, Yoshihiro K, Yoshiko F, Yuko S, Mamoru N, et al. LOX Index, A Novel Predictive Biochemical Marker for Coronary Heart Disease and Stroke. Clin Chem 2010;56:550-8. [Crossref]

22. Noriaki K, Hirokazu M, Kazutaka H, Masaru T, Goro K, Toru K. Soluble Lectin-Like Oxidized LDL receptor-1 (sLOX-1) as A Sensitive and Specific Biomarker for Acute Coronary Syndrome--Comparison with Other Biomarkers. J Cardiol 2010; 56:15965. [Crossref]

23. ZiWen Z, YiWei X, ShuMei L, JinJian G, JianMin S, JuChang H, et al. Baseline Serum sLOX-1 Concentrations Are Associated With 2-Year Major Adverse Cardiovascular and Cerebrovascular Events in Patients After Percutaneous Coronary Intervention. Dis Markers 2019;2019:4925767. [Crossref]

24. Junfeng L, Yunde L, Kegang J, Zhixiao H, Qianyu H, Zhili L, et al. Clinical Analysis of Lectin-Like Oxidized Low-Density Lipoprotein receptor-1 in Patients with in-Stent Restenosis After Percutaneous Coronary Intervention. Medicine (Baltimore) 2018;97:e0366. [Crossref]

25. Andreas S, Antonios K, Stauros S, Maria G, George L, Konstantinos T, et al. Expression of Lectin-Like Oxidized Low-Density Lipoprotein Receptor-1 in Human Epicardial and Intramyocardial Coronary Arteries of Male Patients Undergoing Coronary Artery Bypass Grafting. Cardiology 2018;139:203-7. [Crossref]

26. Nicole S, ChihChieh C, Liang Yin K, ChihSheng C, Jonathan L, Tatsuya S, et al. Interplay between CRP, Atherogenic LDL, and LOX-1 and Its Potential Role in the Pathogenesis of Atherosclerosis. Clin Chem 2016;62:320-7. [Crossref]

27. Jingye D, Hui W, Yuewei W. MiR-98-5p May Be A Biomarker for Screening Bronchial Asthma in Children by Targeting IL-13. Clin Lab 2019; 65. [Crossref]

28. Zhibo C, Mian W, Qiong H, Zilun L, Yang Z, Wenjian W, et al. MicroRNA-98 Rescues Proliferation and Alleviates ox-LDL-induced Apoptosis in HUVECs by Targeting LOX-1. Exp Ther Med 2017;13:1702-10. [Crossref]

29. Chuan S, Huibin L, Jing G, Yang Y, Di Y, Fang H, et al. MicroRNA-98 Negatively Regulates Myocardial Infarction-Induced Apoptosis by Down-Regulating Fas and caspase-3. Sci Rep 2017;7:7460. [Crossref] 